\title{
The effect of tumor size on the imaging diagnosis: A study based on simulation
}

\author{
Huiting Qiao, Libin Wang, Deyu Li, Daifa Wang and Yu Wang* \\ School of Biological Science and Medical Engineering, Beihang University, Beijing 100191, China
}

\begin{abstract}
Positron emission tomography (PET) has been widely used in early diagnosis of tumors. Though standardized uptake value (SUV) is a common diagnosis index for PET, it will be affected by the size of the tumor. To explore how the tumor size affects imaging diagnosis index, dynamic PET images were simulated to study the relationship between tumor size and the imaging diagnosis index. It was found that the SUV of the region of the tumor varied with scan time, and the SUV was always lower than the true value of tumor. Even more deviations were found in SUV with a reduced tumor size. The diagnosis index $\mathrm{SUV}_{\max }$ was more reliable than SUV, for it declined only when the volume of tumor was less than 3 $\mathrm{mm}^{3}$. Therefore, the effect of tumor size on the SUV and $\mathrm{SUV}_{\max }$ that are used as diagnosis indices in the early diagnosis of tumors should not be neglected.
\end{abstract}

Keywords: Standardized uptake value, maximum standardized uptake value, positron emission tomography, tumor size, diagnosis

\section{Introduction}

As one of the most serious threats to life health, cancer is the second leading cause of death in developing countries. The number of people who are diagnosed with cancer and the mortality rates of people diagnosed with malignant tumor are still increasing in recent years [1,2]. It was reported that lung cancer was the leading cause of death in all the common cancer registries [3,4], and its mortality rate increased substantially in the past three years. Although there are some methods of oncologic treatment on clinical therapies, such as radiation, chemotherapy and surgery, it is still hard to improve the survival rate of lung cancer because of late diagnosis $[5,6]$. Therefore, early diagnosis is important to improve treatment and the survival rate of patients with lung cancer [7,8]. Imaging diagnosis [9] as well as identification of molecular biomarkers [10-12] have been used as two main methods in the studies of early diagnosis for lung cancer.

In terms of imaging diagnosis, positron emission tomography (PET) is the function imaging technology that can provide metabolism information of tissues by using a radioactive tracer [13]. Moreover, it is an important tool for staging, prognosis, evaluation of treatment, and, especially, for early diagnosis $[14,15]$. A tumor usually has a higher metabolic rate, so it can be recognized easily by using PET. Besides the visual cancer imaging, PET can also provide some detection indices for diagnosis,

${ }^{*}$ Corresponding author: Yu Wang, School of Biological Science and Medical Engineering, Beihang University, Beijing, China. Tel.: 82338755; Fax: 82315554; E-mail: wangyu@buaa.edu.cn.

0959-2989/14/\$27.50 @ 2014 - IOS Press and the authors. 
treatment, such as total lesion glycolysis (TLG), standardized uptake value (SUV), and maximum standardized uptake value $\left(\mathrm{SUV}_{\max }\right)$ [16].

SUV, which is widely used in the study of cancer, is a semi-quantitative measurement of radioactivity concentration [17]. SUV describes the ratio between the radioactive substances uptake of the lesion and the average uptake of the whole body [18]. SUV and SUV ${ }_{\max }$ are different expressions of SUV. SUV refers to the mean SUV of the region of interest (ROI), while $S_{U V} V_{\max }$ is the maximal SUV in the ROI. SUV and $\mathrm{SUV}_{\max }$ are often used as detection indices for early diagnosis of lung cancer. In order to study the different lung tumors and different study demands, researchers often choose different SUV value. Juhász et al. evaluated metabolic ratio and tracer transport of non-small cell lung cancers by using SUV [19]. Koh et al. studied the relationship between SUV and disease-free survival (DFS) and the relationship between SUV and overall survival (OS). DFS and OS are both evaluation indicators of the prognostic impact of the lung tumor patients receiving chemotherapy after surgery [20]. In Alessandro's study of solitary pulmonary nodule tumor, $\mathrm{SUV}_{\max }$ was used to find significant difference or correlation [21]. Mehta also used $\mathrm{SUV}_{\max }$ to study the research about the patients with non-smallcell lung cancer [16]. In MI B's research [22], SUV=2.5 is used as a threshold value to differentiate benign and malignant lesions. In addition, $\mathrm{SUV}_{\max }$ of normal lung ranges from 1.0 to 1.5 , which can be used as the threshold value to differentiate the normal lung tumor from lung tissue [23]. Thus, SUV and $\mathrm{SUV}_{\max }$ were both studied in this paper as detection indices for early diagnosis of lung cancer.

The SUV is impacted by many factors, such as blood glucose, partial volume effect, body situation, time after injection, and tumor sizes [24].

Although the size of tumor is also an important aspect for SUV calculation and may even cause false negative results, the relationship between the tumor size and the SUV is difficult to study in clinics. Therefore, a mathematical model and simulated PET images were used to study their relationship. The study may be helpful to the imaging diagnosis of lung cancer, and even to that of small size tumors.

\section{Methods}

In order to study the relationship between tumor size and SUV, simulated PET images based on the model were used. First, the chest model with different sizes of tumor was established based on visible human project (VHP) data. Then the dynamic metabolic information was simulated with the metabolic model of tissues, including tumor. And then the dynamic PET images were simulated by mapping techniques. Finally, the SUV and $\mathrm{SUV}_{\max }$ were calculated within the region of interest (ROI) from simulated images. The SUV values from different chest models were compared in term of the sizes of tumor.

\subsection{Chest model}

The chest model was established based on the VHP datasets. VHP provides detailed anatomical information of the human body and it has been widely used in medical imaging segmentation, reconstruction and 3D visualization [25]. The chest model showed in Figure 1 simplifies the chest of the human body through treating some tissues as air, such as fat and the bone of the chest. Lung tumors with eight different sizes were placed in one side of the lung. Each size was tested individually. The chest models using different sizes of tumor are the foundation of the following study. 


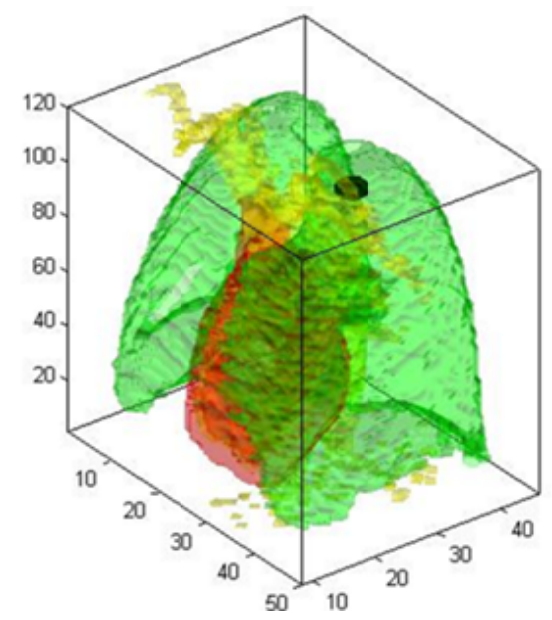

Fig. 1. Schematic diagram of the three-dimensional model.

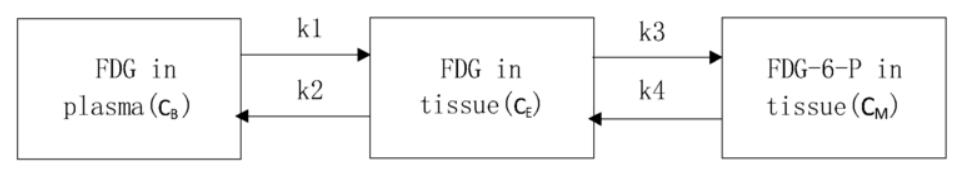

Fig. 2. Three-compartment model.

\subsection{Dynamic metabolic information}

The simulation of the dynamic metabolic information was based on the existed three-compartment model with a $288.566 \mathrm{MBq}$ injected dose every kilogram. The three-compartment model, used in Hang's study of measuring the metabolism ratio of glucose in local brain tissues in 1980 [26], is the most commonly used in the study of FDG-PET ([ $\left.{ }^{18} \mathrm{~F}\right]$ 2-fluoro-2-deoxy-D-glucose, FDG) [27]. Figure 2 shows the structure of the three-compartment model with four parameters.

In the model, three compartments with different concentrations simplify the metabolism system of the human body. $C_{B}$ is the concentration of FDG in blood. $C_{E}$ is the concentration of FDG in tissues. $\mathrm{C}_{\mathrm{M}}$ is the concentration of the phosphorylated FDG-6-P when it came into the cells. The kinetic parameters of the model, $\mathrm{k} 1-\mathrm{k} 4$, express the rate coefficient of the substance exchange in different compartments. The curve, which represents the metabolism of blood changing over time, is called the blood time activity curve (BTAC). As BTAC is the input of this model, it is also called an input curve. The BTAC used in this study is the standard input curve from Feng's article [28]. According to the requirement of the experiment, the sampling protocol is set up as follows: $1 \mathrm{~min} * 8,5 \min * 9$. The time points are $1 \mathrm{~min}, 2 \mathrm{~min}, 10 \mathrm{~min}, 20 \mathrm{~min}, 30 \mathrm{~min}, 40 \mathrm{~min}, 50 \mathrm{~min}$. Table 1 shows the kinetic parameters of different normal tissues (myocardium, lung) which are quoted from Cui's articles [29]. Those of skeletal muscle are quoted from Reinhardt's article [30]. The parameters of the simulated lung tumor are quoted from Torizuka's article on lung tumors [31]. In Table 1, f represents the impact coefficient of surrounding tissues that are affected by the radioactive specific activity of blood [32]. $\mathrm{C}_{\mathrm{T}}$ is defined to represent the output curve, which is also called the tissue time activity curve. 
Table 1

Metabolic model parameters of different organs

\begin{tabular}{llllll}
\hline tissue & \multicolumn{4}{l}{ Model parameters of three-compartment model } \\
\cline { 2 - 6 } name & $\begin{array}{l}\mathrm{K} 1 \\
(\mathrm{ml} / \mathrm{min} / \mathrm{ml})\end{array}$ & $\begin{array}{l}\mathrm{K} 2 \\
(\mathrm{~min}-1)\end{array}$ & $\begin{array}{l}\mathrm{K} 3 \\
(\mathrm{~min}-1)\end{array}$ & $\begin{array}{l}\mathrm{K} 4 \\
(\mathrm{~min}-1)\end{array}$ & $\mathrm{f}$ \\
\hline myocardium & 0.196 & 1.022 & 0.149 & 0.010 & 0.545 \\
lung & 0.014 & 0.291 & 0.006 & 0. & 0.151 \\
lung tumor & 0.231 & 1.149 & 0.259 & 0 & 0 \\
Skeletal muscle & 0.047 & 0.325 & 0.084 & 0 & 0.019 \\
\hline
\end{tabular}

\subsection{Simulated PET images}

TTACs of tissues contain function information of tissues metabolism. VHP provides the detailed anatomical information of the human body. The simulation of PET images combines function information with the anatomical information of the human body. Therefore, the PET images were simulated by using TTACs and VHP.

The method that combines TTACs and VHP data sets is the mapping technique proposed by Cui [33]. Firstly, radioactivity values of tissues at specific time points were extracted by sampling the simulated TTACs. Secondly, these radioactivity values were assigned to the corresponding tissues of the VHP datasets at every time point.

The different sizes of the lung tumors in the simulation are set to $0.5 \mathrm{~mm}^{3}, 1 \mathrm{~mm}^{3}, 2 \mathrm{~mm}^{3}, 3 \mathrm{~mm}^{3}, 5$ $\mathrm{mm}^{3}, 1 \mathrm{~cm}^{3}, 2 \mathrm{~cm}^{3}$, and $3 \mathrm{~cm}^{3}$. The resolution of VHP datasets is $0.33 \mathrm{~mm} * 0.33 \mathrm{~mm} * 1 \mathrm{~mm}$. According to the resolution, the number of lung tumor pixels was calculated. Clinical PET images are commonly datasets with $128 * 128$ pixels in every cross section. However, every layer of VHP datasets has $1760 * 1024$ pixels. In order to keep the proportion of tissues and simulate the partial volume effect, the sample of simulated datasets was dropped by using an average. After dropping the sample of datasets, the final number of pixels in every cross section is $220^{*} 128$. Then the chest models with tumor and dynamic metabolic information were obtained.

\subsection{Calculation of SUV}

SUV (Eq. (1)) represents the ratio of FDG concentration in tissue activity to the injected dose in every kilogram body weight. The SUV is calculated as:

$$
S U V=\frac{\text { tissue activity }(\mathrm{KBq} / \mathrm{ml})}{\text { injected dose }(M B q) / \text { bodyweight }(\mathrm{kg})}
$$

$\mathrm{SUV}_{\max }$ is calculated by using the maximum value of tissue activity instead of the average value of tissue activity. The denominator of the Eq. (1) was estimated as $288.566 \mathrm{MBq} / \mathrm{kg}$, according to a report of Stefani et al. [19]. Tissue activity was obtained by selecting ROI manually from the established chest model. Finally, values of SUV and $\mathrm{SUV}_{\max }$ were calculated by these values and equation. 


\section{Results}

\subsection{Dynamic PET image simulations}

The concentration of FDG in each tissue could be described by TTACs, which were simulated with three-compartment model. The simulated TTACs of myocardium, lung, lung tumor, and skeletal muscle, as well as the standard input curve, are shown in Figure 3. The TTACs show the characters of the metabolism in these four tissues. The difference in metabolism could be used as the basis for the dynamic images segmentation.

Dynamic PET images were simulated by mapping with simulated TTACs. Eight sets of dynamic images were simulated. Various tumor size (from $0.5 \mathrm{~mm}^{3}$ to $3 \mathrm{~cm}^{3}$ ) were included in these eight simulated dynamic images. The dynamic imaging sequences consisted of eight 1-min frames, and nine 5-min frames, totaling 17 frames. Some of the simulated images are shown in Figure 4.

\subsection{The analysis of the simulated dynamic images}

The region of interest, including tumor, were identified on the simulated dynamic images. On this basis, SUV and SUV $\mathrm{max}_{\max }$ of the lung tumors were calculated according to Eq. (1). Figures 5 and 6 show SUV and $\mathrm{SUV}_{\max }$, separately.

Both SUV and SUV ${ }_{\max }$ change along with the time going. In the clinical study, values at $50 \mathrm{~min}$ are the most common used in the diagnosis of tumor. There are differences between changes in SUV and

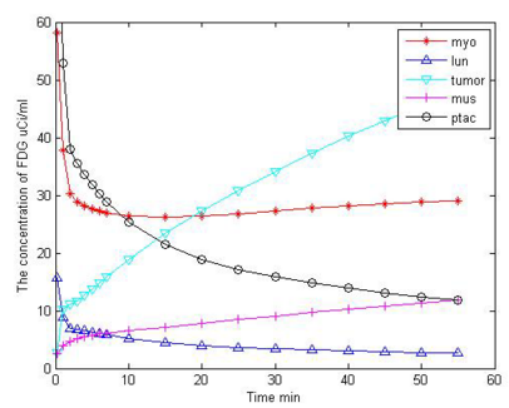

Fig. 3. Simulated TTACs.

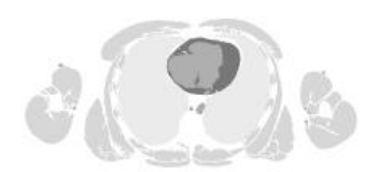

$0.5 \mathrm{~mm}^{3}$

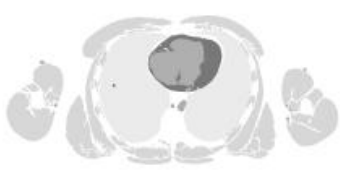

$5 \mathrm{~mm}^{3}$

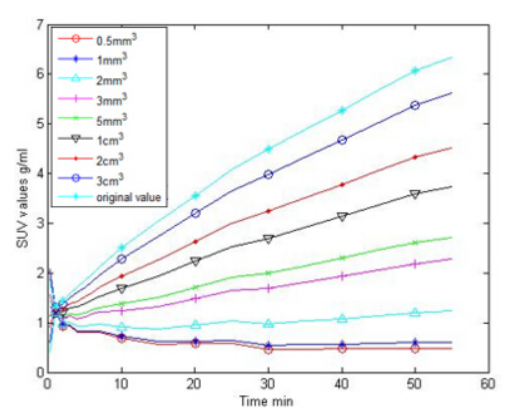

Fig. 5. Comparison between original values and SUV values.

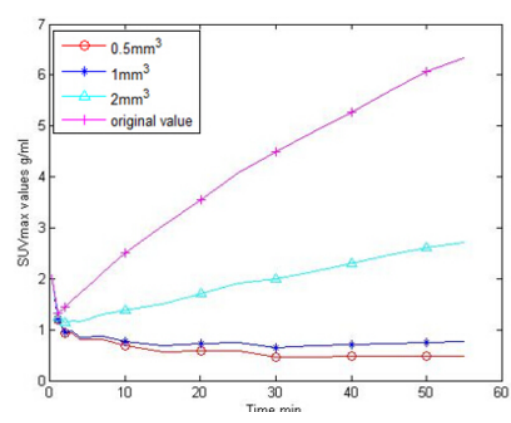

Fig. 6. Comparison of $\mathrm{SUV}_{\max }$ with different sizes tumors.

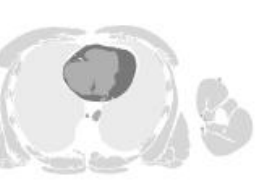

$1 \mathrm{~mm}^{3}$

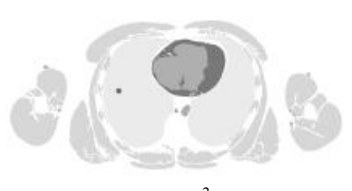

$1 \mathrm{~cm}^{3}$

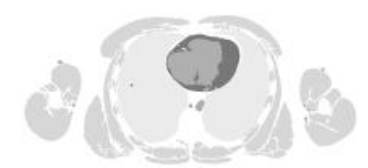

$2 \mathrm{~mm}^{3}$

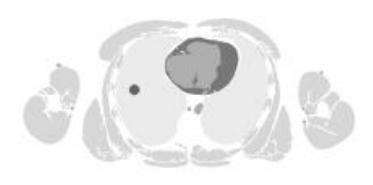

$2 \mathrm{~cm}^{3}$

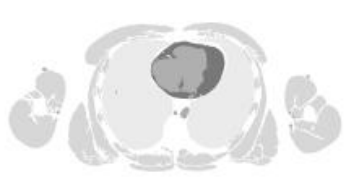

$3 \mathrm{~mm}^{3}$

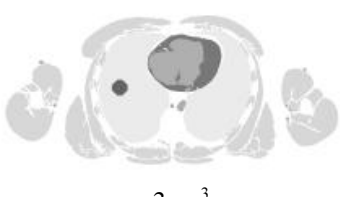

Fig. 4. Simulated PET images. 
$\mathrm{SUV}_{\max }$. SUV values of different tumor size are less than the original values. SUV values decrease along with the decreasing tumor size. $\mathrm{SUV}_{\max }$ of some tumor sizes are equal to the original value and some are less than the original value.

\section{Discussion}

SUV and $\mathrm{SUV}_{\max }$ were studied based on simulated dynamic PET images. It has been certified that $\mathrm{SUV}_{\text {max }}$ is preferred to SUV in tumor diagnosis, as Alessandro et al. [21] reported. The SUV will be affected by the size of the tumor. The effect of tumor sizes was studied by simulating PET images with different sizes tumors. Results of the study show that SUV values change with ongoing monitoring time. $50 \mathrm{~min}$ is the common used time point in the related studies. Because the difference in the tissues is obvious, radioactive concentration of normal tissues tend to be stable at $50 \mathrm{~min}$. However, FDG uptake in the tumor increased over time. SUV can reflect that distribution of the radioactive tracer. Therefore, SUV values at $50 \mathrm{~min}$ were selected to analyze the effect of tumor sizes quantitatively.

Compared with the existing studies, this study focused on the effect of tumor size on diagnosis by using simulated dynamic PET images. Figures 5 and 6 show the relationship between SUV, SUV $_{\max }$ and different tumor sizes, respectively. Figure 5 shows that SUV values increase as time increases. In the simulation study, the SUV values of tumor were lower than the original values for the reason of partial volume effect. SUV values reduce along with the tumor size decreased. In the reference, $\mathrm{SUV}=2.5$ is generally used as a threshold to differentiate benign and malignant lesions. In Figure 5, tumor with sizes $<5 \mathrm{~mm}^{3}$ have SUV values less than 2.5. In other words, the tumor whose size less than $5 \mathrm{~mm}^{3}$ cannot be diagnosed as malignant tumor. Diagnosis of tumor with sizes $<5 \mathrm{~mm}^{3}$ may cause false negative results in the identification of benign and malignant lesions and may result in an inappropriate treatment. $\mathrm{SUV}_{\max }$ values of normal lung range from 1.0 to 1.5 , which are used as a threshold to differentiate the normal lung tumor from lung tissue. As can be seen in Figure 6, $\mathrm{SUV}_{\max }$ values of the tumor with sizes $>2 \mathrm{~mm}^{3}$ are equal to original $\mathrm{SUV}_{\max }$ of tumor. Tumors with sizes $<2$ $\mathrm{mm}^{3}$ have SUV values less than 1.5. In other words, the tumor whose sizes less than $2 \mathrm{~mm}^{3}$ cannot be diagnosed as the tumor. Such results may cause false negative result in the diagnosis of a tumor. The thresholds of study can provide a significant reference for the clinical diagnosis and treatment.

In the clinical study, PET images are affected by noises which were not added in the simulation study. In addition, ROIs were selected manually which caused an error inevitably. These two factors affect the SUV to a certain extent. In a future study, these factors will be studied by adding in the simulated PET images.

\section{Conclusion}

Though SUV is the useful index for tumor diagnosis in PET imaging, it has some limitations. Through the simulation study, tumor size affects the SUV. Tumors whose size is less than $2 \mathrm{~mm}^{3}$ have a SUV $_{\max }$ value of less than 1.5, which lead to a false negative diagnosis of tumor. Tumors, whose size is less than $5 \mathrm{~mm}^{3}$ have a SUV value less than 2.5 , which affect the grade of the tumor. Compared with SUV, SUV $\mathrm{max}_{\max }$ is better than SUV in the aspect of minimizing the partial volume effect. When tumor size is less than $2 \mathrm{~mm}^{3}$, the tumor cannot be diagnosed and evaluated correctly by either SUV or $\mathrm{SUV}_{\max }$. 


\section{Acknowledgement}

This work was supported by National Nature Science Foundation of China (Grant No. 81101123, No. 61108084, No. 61201313, No. 61190125), National Key Technology R \& D Program (Grant No. 2012BAI14B04) and the Fundamental Research Funds for the Central Universities of China.

\section{References}

[1] M.P. Pavlou and E.P. Diamandis, The cancer cell secretome: A good source for discovering biomarkers, Journal of Proteomics 73 (2010), 1896-1906.

[2] W. Cho, Contribution of oncoproteomics to cancer biomarker discovery, Mol. Cancer 6 (2007), 25-27.

[3] W. Chen et al., An analysis of lung cancer mortality in China, 2004-2005, Journal of Preventive Medicine 44 (2010), 378-382.

[4] J. She et al., Lung Cancer in China Challenges and Interventions, Chest 143 (2013), 1117-1126.

[5] H.S. Sekhon et al., Advances in cytopathology for lung cancer: The impact and challenges of new technologies, Thoracic Surgery Clinics 23 (2013), 163-178.

[6] A. Jemal et al., Global cancer statistics, CA: A Cancer Journal for Clinicians 61 (2011), 69-90.

[7] W.C.S. Cho, Molecular diagnostics for monitoring and predicting therapeutic effect in cancer, Expert Review of Molecular Diagnostics 11 (2011), 9-12.

[8] M. Hassanein et al., Advances in proteomic strategies toward the early detection of lung cancer, Proceedings of the American Thoracic Society 8 (2011), 183-188.

[9] Y. Liu and M. Wang, Advances in early diagnosis of lung cancer, Journal of Lung Cancer 14 (2011), 429-434.

[10] L. Cui and H. Wei, Research status and funding trends of lung cancer biomarkers, Journal of Thoracic Disease 5 (2013), 698-705.

[11] Z. Altintas and I. Tothill, Biomarkers and biosensors for the early diagnosis of lung cancer, Sensors and Actuators BChemical 188 (2013), 988-998.

[12] C.H. Oak et al., Potential molecular approaches for the early diagnosis of lung cancer (Review), Molecular Medicine Reports 6 (2012), 931-936.

[13] S. Thureau et al., Interobserver Agreement of qualitative analysis and tumor delineation of f-18-fluoromisonidazole and 3 '-deoxy-3 '-f-18-fluorothymidine PET images in lung cancer, Journal of Nuclear Medicine 54 (2013), 1543-1550.

[14] N. Avril et al., Response to therapy in breast cancer, Journal of Nuclear Medicine 50 (2009), 55-63.

[15] L. Schrevens et al., The role of PET scan in diagnosis, staging, and management of non-small cell lung cancer, Oncologist 9 (2004), 633-643.

[16] G. Mehta et al., Feasibility study of FDG PET/CT-derived primary tumor glycolysis as a prognostic indicator of survival in patients with non-small-cell lung cancer, Clinical Radiology 69 (2014), 268-274.

[17] O. Demirkaya, Lesion segmentation in whole-body images of PET, Nuclear Science Symposium Conference Record 4 (2003), 2873-2876.

[18] K.R. Zasadny and R.L. Wahl, Standardized uptake values of normal tissues at PET with 2-fluorine-18-fluoro-2-deoxyD-glucose: Variations with body weight and a method for correction, Radiology 189 (1993), 847-850.

[19] C. Juhasz et al., Quantification of tryptophan transport and metabolism in lung tumors using PET, Journal of Nuclear Medicine 50 (2009), 356-363.

[20] Y. Koh et al., EGFR gene copy number gain is related to high tumor SUV and frequent relapse after adjuvant chemotherapy in resected lung adenocarcinoma, Journal of Clinical Oncology 41 (2011), 548-554.

[21] A. Stefani et al., Integrated FDG-PET/CT imaging is useful in the approach to carcinoid tumors of the lung, Journal of Cardiothoracic Surgery 8 (2013), 1-7.

[22] B. MI et al., The value of extra-lung lesions on 18 F-FDG PET/CT in improving diagnosis of lung cancer, Chinese Journal of Lung Cancer 15 (2012), 78-83.

[23] C.D. Ramos et al., FDG-PET standardized uptake values in normal anatomical structures using iterative reconstruction segmented attenuation correction and filtered back-projection, Journal of Nuclear Medicine 28 (2001), 155-164.

[24] P. Obara and Y. Pu, Prognostic value of metabolic tumor burden in lung cancer, Chinese Journal of Cancer Research, 25 (2013), 615-622.

[25] A. Pommert et al., Creating a high-resolution spatial/symbolic model of the inner organs based on the visible human, Medical Image Analysis 5 (2001), 221-228. 
[26] S.C. Huang et al., Noninvasive determination of local cerebral metabolic rate of glucose in man, The American Journal of Physiology 238 (1980), 69-82.

[27] Y.T. Hong and T.D. Fryer, Kinetic modelling using basis functions derived from two-tissue compartmental models with a plasma input function, Neuroimage $\mathbf{5 1}$ (2010), 164-172.

[28] D. Feng et al., A technique for extracting physiological parameters and the required input function simultaneously from PET image measurements: theory and simulation study, IEEE Transactions on Information Technology in Biomedicine: A Publication of the IEEE Engineering in Medicine and Biology Society 1 (1997), 243-254.

[29] Y. Cui et al., Parameter estimation for whole-body kinetic model of FDG metabolism, Progress in Natural Science $\mathbf{1 6}$ (2006), 1164-1170.

[30] M. Reinhardt et al., Quantification of glucose transport and phosphorylation in human skeletal muscle using FDG PET, Journal of Nuclear Medicine 40 (1999), 977-985.

[31] T. Torizuka et al., Untreated primary lung and breast cancers: correlation between F-18 FDG kinetic rate constants and findings of in vitro studies, Radiology 207 (1998), 767-774.

[32] X. Li et al., Estimation of myocardial glucose utilisation with PET using the left ventricular time-activity curve as a non-invasive input function, Medical and Biological Engineering and Computing 36 (1998), 112-117.

[33] Y.F. Cui and J. Bai, Method of simulation and visualization of FDG metabolism based on VHP image, Medical Imaging 2005: Visualization, Image-Guided Procedures, and Display, Pts. 1 and 25744 (2005), 547-554. 